\title{
Spectral Detection of Metal Contaminants in Water Using an On-Chip Microglow Discharge
}

\author{
Chester G. Wilson and Yogesh B. Gianchandani, Member, IEEE
}

\begin{abstract}
This paper reports on the detection of trace contaminants in water by spectroscopy of micro glow discharges that operate in air or at moderate vacuum using liquid electrodes. A liquid $e$ lectrode spectral $e$ mission $c$ hip (LEd-SpEC) has been developed to perform this function. The device is fabricated by a four mask process, and provides a reservoir and channels in a glass substrate, along with electrodes that bias the water sample. Liquid from the cathode is sputtered into the discharge, for spectroscopic detection of impurities. Using a commercial spectrometer, Na concentrations $<10 \mathrm{ppm}$, and $\mathrm{Pb}$ concentrations of $5 \mathrm{ppm}$, and $\mathrm{Al}$ and $\mathrm{Cr}$ concentrations of $10 \mathrm{ppm}$ have been measured. The ratio of Na spectral intensity to that of ambient $\mathrm{N}_{2}$ is shown to be a suitable measure of $\mathrm{Na}$ impurity concentration over several orders of magnitude. The addition of $\mathrm{HNO}_{3}$ to lower the $\mathrm{pH}$ of the liquid solution increases this ratio by almost an order of magnitude. By selectively doping the solution, the device can also be used as a customizable optical source for UV and visible wavelengths.
\end{abstract}

Index Terms-Chemical detection, microdischarge, microplasma, water spectroscopy.

\section{INTRODUCTION}

W ITH threats to potable water sources from industrial and biochemical pollutants, diagnostic tools that can provide rapid on-site tests for contaminants are of significant interest. Presently, water quality assessment is a relatively elaborate process, requiring sample transportation and laboratory analysis at centralized locations. Analysis involves the use of a plasma spectrometer, which varies in size from a table top model to room size. The water sample is sprayed into a radio frequency (RF) inductively coupled plasma, and the optical emission is analyzed to determine the composition and quantity of the water impurities. This is similar to detection of gaseous species in plasmas.

A variety of methods are available for diagnosis of gas and water impurities and several of these are being explored for microsystems applications. For analyzing liquids, FET structures with ion-sensitive gate material are used [1]. Gas chromatographs separate different gases flowing through a heated tube together with a carrier gas, on the basis of their sticking coefficients to the heated wall. Recent efforts have been made

Manuscript received June 3, 2002. This work was supported in part by a gran from the U.S. Geological Survey through the Water Resources Institute and by a grant from the University of Wisconsin Sea Grant Institute. The review of this paper was arranged by Editor K. Najafi.

C. G. Wilson is with the Department of Electrical and Computer Engineering, University of Wisconsin, Madison WI 53706 USA (e-mail: chwilsn@engin.umich.edu).

Y. B. Gianchandani is with the University of Michigan, Ann Arbor, MI 48109 USA (e-mail: yogesh@umich.edu).

Digital Object Identifier 10.1109/TED.2002.805608

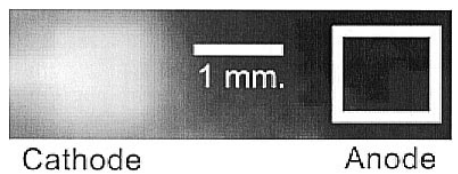

Fig. 1. Local confinement of a microplasma at 2 Torr and $410 \mathrm{~V}$ dc.

to miniaturize these [2]. Kelvin cell gas detectors have been micromachined [3]. These devices measure the change in the work function of a solid surface as it absorbs gas.

Devices that measure impurities by ionizing the sample and measuring the spectra have the potential for very sensitive measurements, and the advantages of a large database of spectral characteristics. Mass spectrometers, which measure the ratio of ion mass to charge are often used for detecting gases. In particular, quadrupole mass spectrometers have an ion source, an electrostatic lens with four poles to focus the ion flow, and an array of detectors to measure the spatial deflection of ions, which is proportional to their charge to mass ratios. Ions with smaller mass to charge ratios are deflected more. Using micromachining technology, a quadrupole mass spectrometer has been developed with 500- $\mu \mathrm{m}$ diameter electrodes [4]. Time of flight mass spectrometers ionize gas atoms in bursts, accelerate the ions electrostatically, and measure their time of flight, which is a function of ion mass. Ion mobility mass spectrometers, which have the capability of operating at atmospheric pressure, exploit differences in the mobilities of species in the presence of a background gas. An RF ion mobility spectrometer has recently been miniaturized [5].

Microplasmas have been the focus of increasing research in recent years. We have reported through-wafer etching of a silicon substrate using dc microplasmas which were ignited across a metal-polyimide-metal electrode stack patterned on the same substrate [6]. In contrast to the stacked electrode configuration, a configuration that uses two coplanar titanium electrodes is shown in Fig. 1. This particular microplasma was generated in air at 2 Torr, at $410 \mathrm{~V}$. The glow in these coplanar electrode microplasmas is confined to the region over the cathode. Other efforts have been directed at miniaturizing inductively coupled plasmas to be utilized for gas spectroscopy [7], and to utilize dc microplasmas as an optical emission source for gas chromatography [8]. Atomic transitions of metallic impurities are typically best detected from spectroscopic analysis of dc plasma emissions [9]. Work has also been done employing the water sample as a cathode, with a metallic anode for spectroscopic use [10], [11], and detecting water impurities through spectral information. An effort to implement this system in a MEMS device has been reported very recently [12]. 


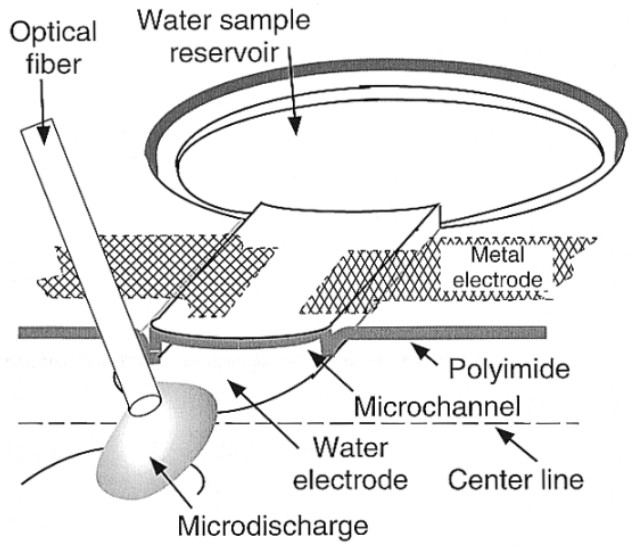

Fig. 2. Schematic of device concept showing half of the symmetrical structure.

This paper reports on the detection of trace contaminants in water by spectroscopy of micro glow discharges that operate in air using dual liquid electrodes (i.e., both the anode and cathode are liquid). A liquid electrode spectral emission chip (LEd-SpEC) has been developed to perform this function. ${ }^{1}$ Liquid from the cathode is sputtered into the discharge, and can be utilized for spectroscopic detection of impurities such as $\mathrm{Pb}, \mathrm{Cr}$, and others. This device can also be used as a customizable optical source for UV and visible wavelengths. The device structure and operation are described in Section II. Section III describes the fabrication of the device, and the experimental results are shown in Section IV.

\section{Device Structure}

The LEd-SpEC uses the water sample, inserted into microchannels on a glass substrate, as the electrode material for generating a glow discharge for spectroscopic analysis (see Fig. 2). This provides the following benefits:

1) the water sample and its impurities are effectively sputtered into the glow discharge, eliminating the need for spraying, which is otherwise necessary for detecting nonvolatile contaminants;

2) concern for electrode wear is eliminated since it is replenished by liquid flow;

3) a continuous glow discharge can be sustained in air at atmospheric pressure, eliminating the need for a vacuum system.

Thin-film Pt features patterned within the channel provide lead transfers for the dc power supply. A 4.5-mm long channel covered by polyimide separates the exposed liquid in the $500 \mu \mathrm{m} \times$ $700 \mu \mathrm{m}$ electrode regions from reservoirs, which do not participate in the glow discharge. The two liquid electrodes in each device are separated by $2.5 \mathrm{~mm}$. An optical fiber mounted above this region couples the device to a pager-sized spectrometer (see Fig. 3) (Ocean Optics USB-2000) connected to a data acquisition computer. (The LEd-SpEC can accommodate a copackaged micro spectrometer as well.) [14].

${ }^{1} \mathrm{~A}$ portion of this work has been published in conference abstract form in

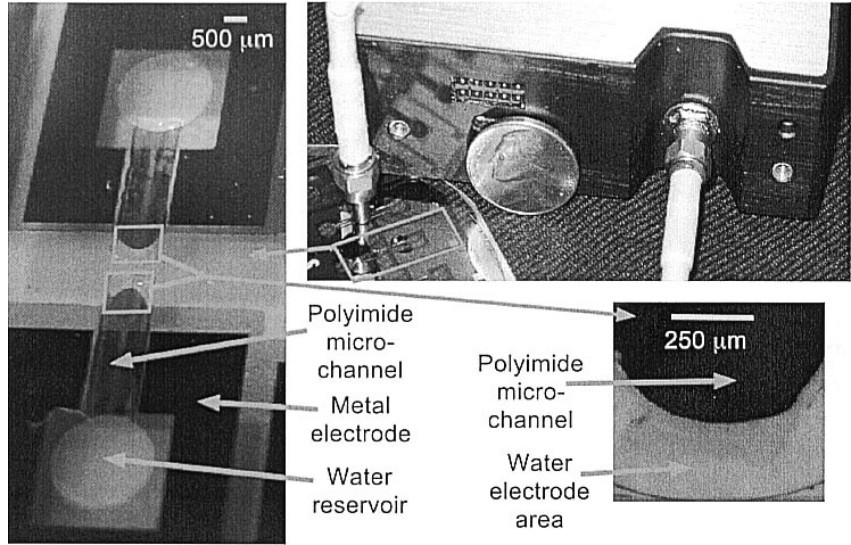

Fig. 3. Optical viewgraph of system and close-up view of the LEd-SpEC.

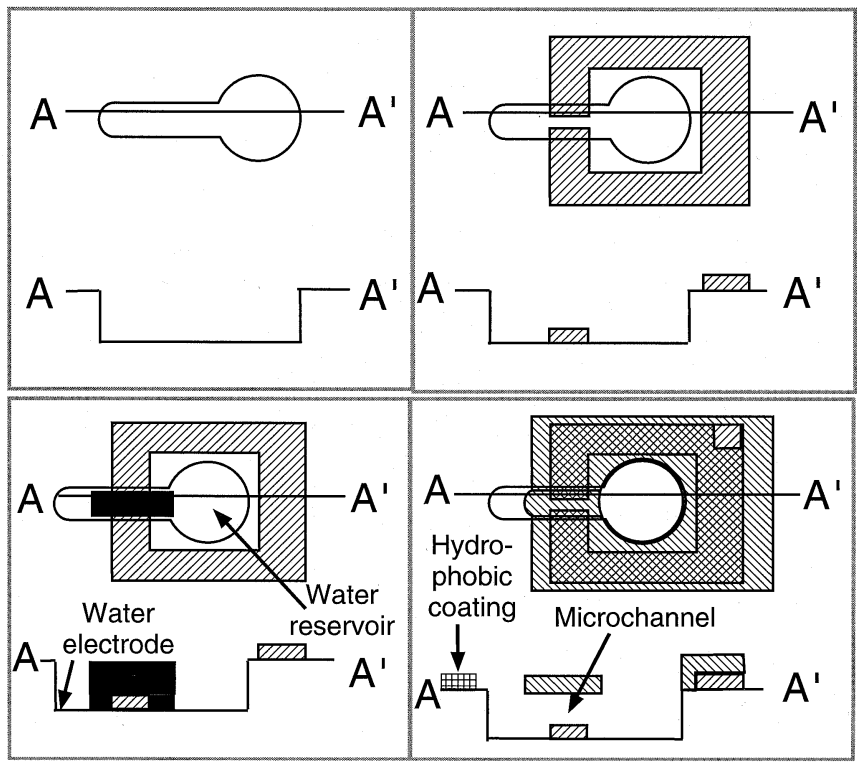

Fig. 4. Fabrication sequence: 1) recess glass substrate; 2) pattern Pt lead transfer; 3) electroplate $\mathrm{Cu}$ sacrificial layer for channel; and 4) pattern polyimide cover, etch sacrificial layer, and apply hydrophobic film between electrodes.

\section{FABRICATION}

The device is fabricated on a glass substrate using a 4-mask process (see Fig. 4). The first mask serves to pattern an etch in a glass wafer which serves to define the sample confinement region. In this step, a 300/800 $\AA \mathrm{Cr} / \mathrm{Au}$ layer is evaporated onto the glass substrate, and patterned by lift-off. The exposed glass substrate is wet-etched using a 3:5:7 HF/Nitric acid/DI water solution, following which the $\mathrm{Cr} / \mathrm{Au}$ layer is removed. The second mask patterns thin-film Pt electrodes, which provide electrical contact for the liquid electrodes. These electrodes are formed by evaporating a 300/500 $\AA \mathrm{Cr} / \mathrm{Pt}$ layer which is also patterned by lift-off. Platinum was chosen as the electrode material, because it is unreactive with most acidic solutions. The third mask provides a photoresist mold for a 30- $\mu$ m thick layer of electroplated $\mathrm{Cu}$, which is used as a sacrificial layer. A $300-\AA \mathrm{Cu}$ seed layer is first evaporated onto the sample. Photoresist is slow-spun onto the sample to 30- $\mu \mathrm{m}$ thickness, and the $\mathrm{Cu}$ sacrificial layer exposed. Copper is electroplated into the mold in a commercial 


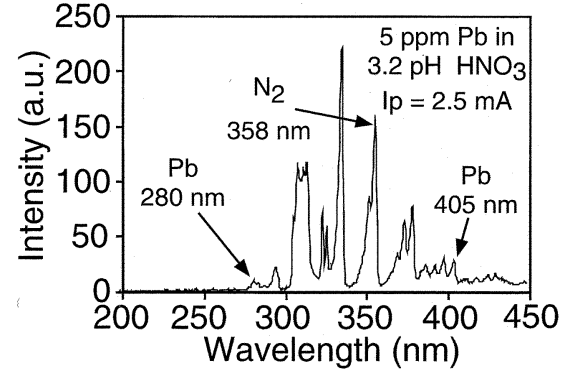

Fig. 5. Spectrum from sample containing $5 \mathrm{ppm} \mathrm{Pb.}$

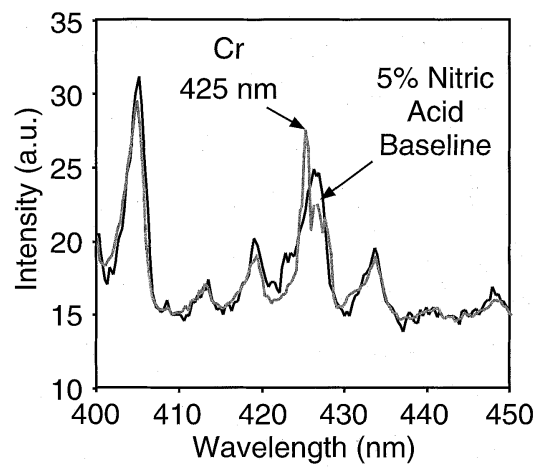

Fig. 6. Spectrum from sample containing $10 \mathrm{ppm}$ chrome and 5\% nitric acid, superimposed against a $5 \%$ nitric acid baseline.

room temperature copper sulfate plating solution at $20 \mu \mathrm{m} / \mathrm{h}$. After electroplating the photoresist is stripped and the $\mathrm{Cu}$ briefly etched to remove regions of the $\mathrm{Cu}$ seed layer that was not electroplated. A 9- $\mu \mathrm{m}$ thick polyimide is then spun on the sample, and is patterned with the fourth mask. This mask provides wet etched openings in a 200/500 $\AA \mathrm{Al} / \mathrm{Ti}$ sputtered layer. The polyimide is then plasma etched with an $100 \mathrm{sccm} \mathrm{O}_{2} / 10 \mathrm{sccm} \mathrm{CF} 4$ gas flow mixture, operated at $500 \mathrm{~W}$ power, and $200 \mathrm{mTorr}$ operating pressure. This patterning exposes the openings in the microchannels, and provides polyimide walls for the sample reservoir. Finally, the sacrificial $\mathrm{Cu}$ is etched away with heated ferric chloride solution, and a hydrophobic film of petroleum jelly is applied to the inter-electrode surface to eliminate electrophoresis of water during prolonged operation [13].

\section{EXPERIMENTAL RESULTS}

Fig. 5 shows the typical spectral output of a sample containing $5 \mathrm{ppm} \mathrm{Pb}$ dissolved in nitric acid with a $\mathrm{pH}$ of 3.2. Two dominant spectral lines characteristic to $\mathrm{Pb}$ are seen at 280 and at $405 \mathrm{~nm}$. Fig. 6 shows the spectral output of a sample containing $10 \mathrm{ppm}$ $\mathrm{Cr}$, contained in a 5\% nitric acid solution. The spectra is shown against a baseline spectra generated from $5 \%$ nitric acid only. The principal spectra due to $\mathrm{Cr}$ at $425 \mathrm{~nm}$ can be seen, as well as some lesser lines in the spectral region. Fig. 7(a) illustrates the spectra generated from $10 \mathrm{ppm} \mathrm{Al} \mathrm{in} \mathrm{5 \%} \mathrm{nitric} \mathrm{acid,} \mathrm{whereas}$ Fig. 7(b) illustrates the spectra generated from 5\% nitric acid only. Fig. 7(a) shows the dominant spectral line at $396 \mathrm{~nm}$ due to $\mathrm{Al}$, as well as a lesser line at $360 \mathrm{~nm}$.

Although the intent of this effort is to permit detection of toxic metal impurities in water, a number of studies that provide basic device characterization were performed with $\mathrm{Na}$, which is
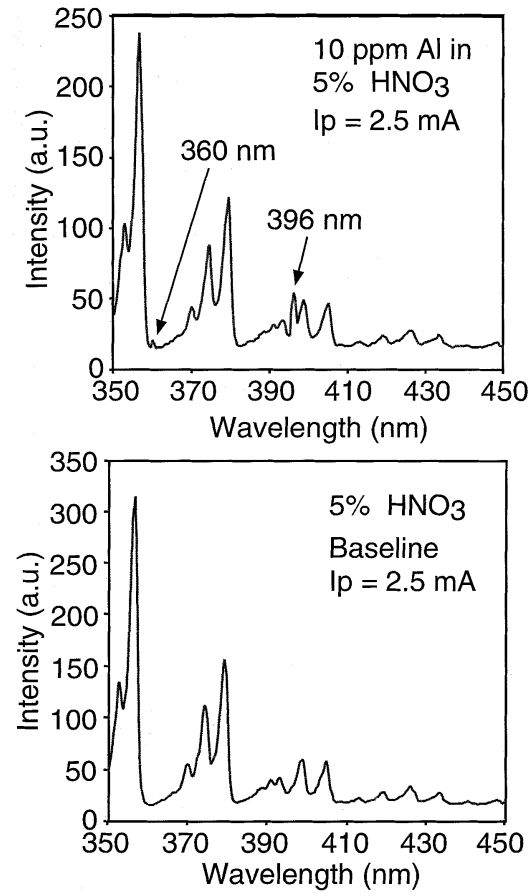

Fig. 7. (Top) Spectrum from sample containing $10 \mathrm{ppm} \mathrm{Al} \mathrm{in} \mathrm{a} \mathrm{5 \%} \mathrm{nitric} \mathrm{acid}$ solution and (bottom) 5\% nitric acid baseline.

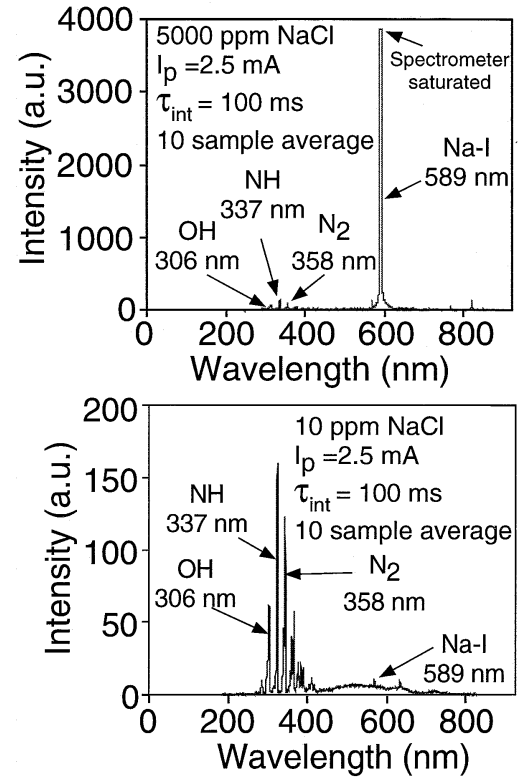

Fig. 8. Spectra for $\mathrm{NaCl}$ samples with concentrations of (top) $5000 \mathrm{ppm}$ and (bottom) $10 \mathrm{ppm}$.

readily available, nontoxic, and provides a robust spectral intensity. Spectra of saline at $10 \mathrm{ppm}$ to $5000 \mathrm{ppm}$ concentrations are shown in Fig. 8. A discharge current of $2.5 \mathrm{~mA}$ was used. These show the primary $\mathrm{Na}$ contaminant line at $589 \mathrm{~nm}$. The large spike at $358 \mathrm{~nm}$ is characteristic to $\mathrm{N}_{2}$. The $\mathrm{Na}$ spectra are a result of the impurities sputtered from the liquid electrode, while the $\mathrm{N}_{2}$ spectra which are seen in all discharges in this effort, are due to the air ambient. The $\mathrm{NH}$ and $\mathrm{OH}$ ionic lines are also visible as a result of the sputtered water, and its reaction with the atmosphere. In Fig. 8(a), the case of $5000 \mathrm{ppm} \mathrm{Na}$, the 


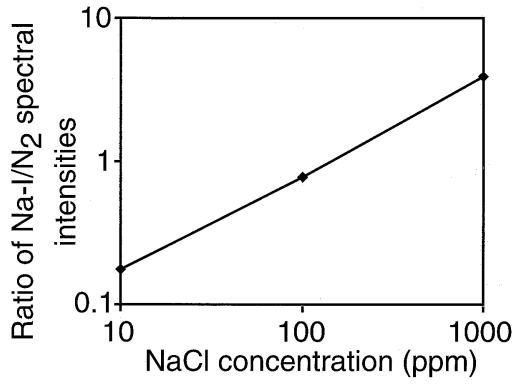

Fig. 9. Ratio of $\mathrm{Na}$ to $\mathrm{N}_{2}$ spectral intensities as a function of $\mathrm{NaCl}$ concentration.

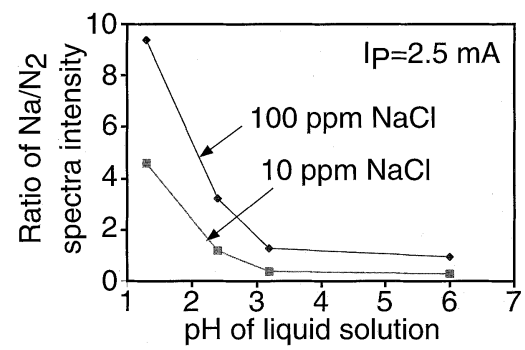

Fig. 10. Ratio of $\mathrm{Na}$ to $\mathrm{N}_{2}$ spectral intensities as a function of $\mathrm{pH}$ of the tested solution.

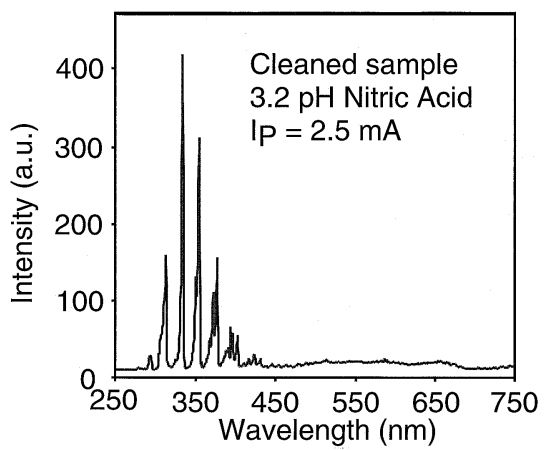

Fig. 11. Spectrum from device previously containing $5000 \mathrm{ppm} \mathrm{NaCl}$, rinsed with DI water.

sodium spectral intensity is so strong the spectrometer is saturated at the Na wavelength, and the signal is much stronger than the $\mathrm{N}_{2}$ lines. In Fig. 8(b), the case of $10 \mathrm{ppm} \mathrm{Na}$, the $\mathrm{N}_{2}$ lines dominate the $\mathrm{Na}$ lines.

By measuring the ratio of the spectral intensities of the primary contaminant to $\mathrm{N}_{2}$, is it possible to determine the concentration of the impurity over a wide dynamic range. This is a valuable feature. Fig. 9 shows the averaged ratio of $\mathrm{Na}$ to $\mathrm{N}_{2}$ spectra, for Na concentration ranging from $10 \mathrm{ppm}$ to $1000 \mathrm{ppm}$. The ratio of spectral intensities varies by almost two orders of magnitude.

The intensity of the Na line can be increased by modifying the $\mathrm{pH}$ of the sample under test with nitric acid, while the intensity of the nitrogen line remains relatively unchanged. This results in the ratio of the $\mathrm{Na}$ to $\mathrm{N}_{2}$ spectral intensity lines to increase as the $\mathrm{pH}$ is decreased, which can be useful for extending the impurity detection limits (see Fig. 10).

LEd-SpEC is designed to be a disposable device, but it can also be reused. Fig. 11 shows deionized water spectra from the same device that was previously used for $5000 \mathrm{ppm} \mathrm{NaCl}$ in

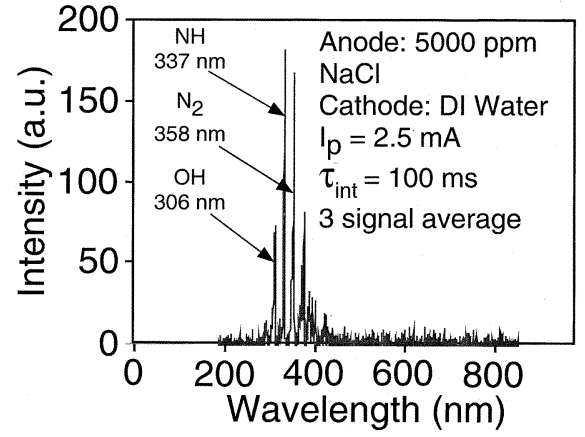

Fig. 12. Spectrum from experiment showing that all contamination measured is from cathode cell.

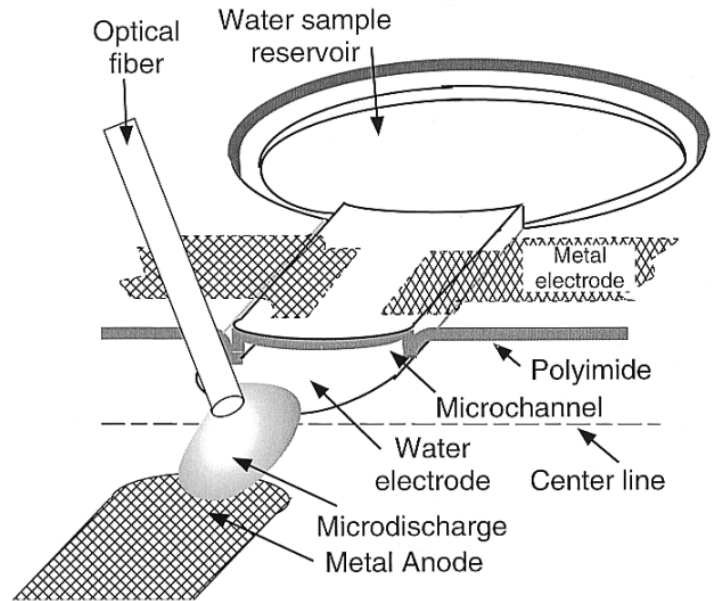

Fig. 13. Schematic of device concept using metal anode.

Fig. 8(a), and was then rinsed in DI water. No sodium spectra can be detected after cleaning.

To determine the source of the impurity ions in the glow discharge, the cathode of one device was filled with a $5000 \mathrm{ppm}$ $\mathrm{NaCl}$ solution and the anode with DI water. This provided a spectrum similar to Fig. 8(a), indicating that positive gas ions from the discharge sputtered the cathode. The spectrum obtained under the reverse polarity did not show the Na line (Fig. 12). This indicates that the dominant impurity delivery mechanism is sputtering from the cathode as opposed to fluid heating and vaporization. This is an important feature of the LEd-SpEC because it permits inorganic impurities which are nonvolatile to be introduced into the plasma. It eliminates the need for spraying the water into the plasma, which is the approach used in conventional devices.

Since it has been verified that the impurity delivery is due to sputtering from the cathode, the device can be constructed with a metal anode, as opposed to a liquid one (see Fig. 13). The dual water electrode configuration allows one reservoir to be filled with the solution under test, and the other reservoir to be filled with a baseline solution, while the metal anode configuration provides a simpler device. The metal anode configuration also provides a lower breakdown voltage. The device is able to detect impurities with sensitivities comparable to the liquid anode device. The spectra created from a solution with $100 \mathrm{ppm} \mathrm{NaCl}$ are shown in Fig. 14. The impact of varying the inter-electrode gap was tested using $5000 \mathrm{ppm} \mathrm{NaCl}$ on device variants (see 


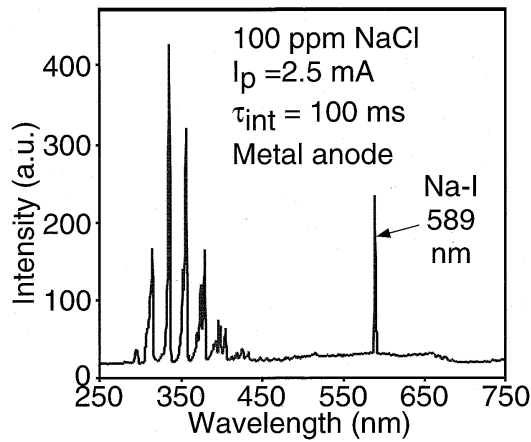

Fig. 14. Spectra generated from device with metal anode, and cathode filled with $100 \mathrm{ppm} \mathrm{NaCl}$.

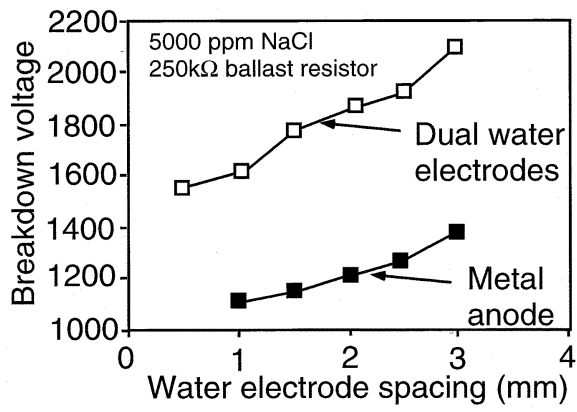

Fig. 15. Breakdown voltage dependence on inter-electrode gap for $5000 \mathrm{ppm}$ $\mathrm{NaCl}$ for dual liquid electrode device, and metal anode device.

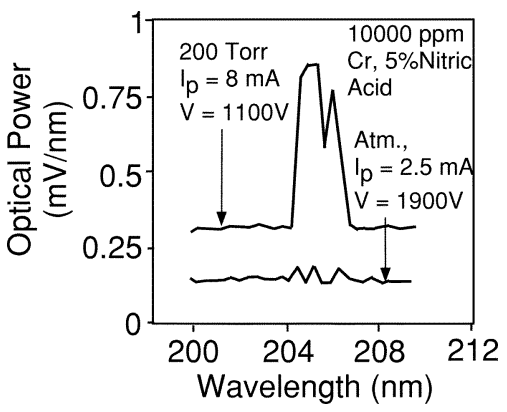

Fig. 16. Spectral intensity characteristics of LEd-SpEC with cathode filled with $10000 \mathrm{ppm}$ chrome, at atmosphere and 200 Torr.

Fig. 15). Results showed a linear relationship between this dimension and the breakdown voltage, with the metal anode configuration providing a lower breakdown voltage.

In addition to use in the detection of water impurities, the LEd-SpEC shows promise as a light source with a customizable spectrum. The device was tested with a $10000 \mathrm{ppm}$ Cr solution in $5 \%$ nitric acid. Cr provides multiple dominant spectral lines in the 200-207 nm range. It was found that the emission spectral power of the UV range increases if the device is operated in vacuum. Fig. 16 illustrates the spectral optical intensity both at atmosphere, and at 200 Torr in air. The intensity is found to increase at lower operating pressures. This is believed to be due to the increased electron energy in discharges at lower operating pressures.

\section{CONCLUSIONS}

The LEd-SpEC device was shown to detect sodium impurities of concentration less than $10 \mathrm{ppm}$, lead impurities at $5 \mathrm{ppm}$, and aluminum and chrome impurities of $10 \mathrm{ppm}$. The device operates by sputtering the cathode, which is the liquid sample under test. The relative concentration of sodium is a function of the ratio of the $\mathrm{Na} / \mathrm{N}_{2}$ spectral intensities, and varies over orders of magnitude. The addition of acid to lower the $\mathrm{pH}$ of the sample increases the intensity of the impurity line. The device can be operated with a metal or liquid anode, and is reusable. The device shows promise as a possible on chip UV as well as optical source.

\section{ACKNOWLEDGMENT}

The authors would like to thank Prof. M. Zorn of the University of Wisconsin-Green Bay, and Prof. M. Anderson and Dr. W. Zeltner of the University of Wisconsin-Madison for helpful discussions. The authors would also like to thank $\mathrm{R}$. Toonen for initial efforts in the project.

\section{REFERENCES}

[1] J. Yoon, D. K. Lee, H. Nam, G. S. Cha, T. D. Strong, and R. B. Brown, "Ion sensors using one-component room temperature vulcanizing-type silicone rubber matrices," J. Electroanal. Chem., vol. 464, pp. 135-142, 1999.

[2] C. M. Yu, M. Lucas, J. C. Koo, P. Stratton, T. DeLima, and E. Behymer, "A high performance hand-held gas chromatograph," in Proc. Micro Electro Mechanical Systems ASME Int. Mechanical Engineering Congress and Exposition, 1998, pp. 481-486.

[3] P. L. Bergstrom, S. V. Patel, J. W. Schwank, and K. D. Wise, "A micromachined surface work-function gas sensor for low-pressure oxygen detection," Sens. Actuators B, Chem., vol. 42, pp. 195-204, Aug. 1997.

[4] S. Taylor, B. Srigengan, J. R. Gibson, D. Tindall, R. Syms, T. J. Tate, and M. M. Ahmad, "A miniature mass spectrometer for chemical and biological sensing," in Proc. SPIE, 2000, pp. 187-193.

[5] R. A. Miller, E. G. Nazarov, G. A. Eiceman, and A. T. King, "A MEMS radio-frequency ion mobility spectrometer for chemical vapor detection," Sens. Actuators A, Phys., vol. 91, pp. 301-12, July 2001.

[6] C. G. Wilson and Y. B. Gianchandani, "Silicon micromachining using in situ DC microplasmas," J. Microelectromech. Syst., vol. 10, pp. 50-54, Mar. 2001.

[7] J. A. Hopwood, "A microfabricated inductively coupled plasma generator," J. Microelectromech. Syst., vol. 9, pp. 309-13, Sept. 2000.

[8] J. C. T. Eijkel, H. Stoeri, and A. Manz, "A DC microplasma on a chip employed as an optical emission detector for gas chromatography," Anal. Chem., vol. 72, pp. 2547-2552, June 2000.

[9] N. W. Routh, "DCP advantages," Appl. Res. Lab. Applicat. Rep.

[10] T. Cserfalvi and P. Mezei, "Emission studies on a glow-discharge in atmospheric air using water as a cathode," J. Phys. D, Appl. Phys., vol. 26, pp. 2184-2188, 1993.

[11] R. K. Marcus and W. C. Davis, "An atmospheric pressure glow discharge optical emission source for the direct sampling of liquid media," Anal. Chem., vol. 73, pp. 2903-2910, 2001.

[12] G. Jenkins and A. Manz, "Optical emission detection of liquid analytes using a micro-machined DC glow discharge device at atmospheric pressure," in Proc. Micro Total Analysis Systems, 2001, pp. 349-350.

[13] K. Handique, D. T. Burke, C. H. Mastrangelo, and M. A. Burns, "Nanoliter-volume discrete drop injection and pumping in microfabricated chemical analysis systems," in Proc. Solid State Sensors and Actuators Workshop, Hilton Head, SC, 1998, pp. 346-349.

[14] C. G. Wilson and Y. B. Gianchandani, "LEd-SpEC: Spectroscopic detection of water contaminants using glow discharges from liquid electrodes," in Proc. IEEE Int. Conf. MEMS, 2002, pp. 248-51. 
Chester G. Wilson received the B.S. degree in electrical engineering and the M.S. degree in applied physics from Washington University, St. Louis, MO, in 1993 and 1996, respectively. He is currently pursuing the Ph.D. degree in electrical engineering at the University of Wisconsin, Madison, in the field of MEMS.

He has studied topics in plasmas and microfabrication focusing on techniques to scale down plasmas into microplasmas, for practical use in silicon etching, chemical analysis, and to study their physical properties.

Mr. Wilson received the 2000-2001 Intel Foundation Graduate Fellowship Award, and the 2002 Gerald Holdbridge Best Student Paper Award.
Yogesh B. Gianchandani (S'92-M'95) received the B.S., M.S., and Ph.D. degrees in electrical engineering in 1984, 1986, and 1994, respectively.

$\mathrm{He}$ is presently with the EECS Department at the University of Michigan, Ann Arbor. Prior to this, he was with the ECE Department at the University of Wisconsin, Madison. He has also held industry positions with Xerox Corporation, Microchip Technology, and other companies, working in the area of integrated circuit design. His research interests include all aspects of design, fabrication, and packaging of micromachined sensors and actuators and their interface circuits. He serves on the editorial boards of Sensors and Actuators, IOP Journal of Micromechanics and Microengineering, and Journal of Semiconductor Technology and Science.

Prof. Gianchandani received the National Science Foundation Career Award in 2000. He also serves on the steering and technical program committees for the IEEE International Conference on Microelectro Mechanical Systems (MEMS), and served as the General Co-chair for this meeting in January 2002. 